\title{
Mental Connection at Distance: Useful for Solving Difficult Tasks?
}

\author{
Patrizio E. Tressoldi, Stefano Massaccesi, Massimiliano Martinelli, Sara Cappato \\ Dipartimento di Psicologia Generale, Università di Padova, Padova, Italy. \\ Email: patrizio.tressoldi@unipd.it \\ Received August 19 $9^{\text {th }}, 2011$; revised September 23 ${ }^{\text {rd }}, 2011$; accepted October $25^{\text {th }}, 2011$.
}

\begin{abstract}
Aim of this study is to provide a demonstration of the non-local property of the human mind to connect at distance, that is, without the classical means of communication. In the first experiment, 40 participants were requested to identify in two separate sessions, 10 real and 10 false Chinese ideograms presented randomly, trying to connect mentally with the research assistant sending correct suggestions at distance that is without any possibility to communicate with them by conventional means. As control condition, in one of these two sessions the helper did not send any suggestion although the receiver believed the contrary. In the session without suggestion, the hits' mean score was 10.55; conversely, in the condition where a research assistant tried to suggest the correct identification at distance, the hits' mean score was 11.33. Both a frequentist and a Bayesian statistical analysis approach, allows to reject the Null Hypothesis supporting the alternative one, that is, the possibility of mental connection at distance exploiting the non-local properties of the human mind. A second experiment aimed at increasing the efficiency of this mental connection taking into account task complexity and the level of Absorption of participants as a personality trait deemed favorable to non-local communication. However the results were similar to the first experiment. Although mental connection at distance seems feasible, variables which positively moderate this kind of communication are still to be identified.
\end{abstract}

Keywords: Non-Local Mental Connection, Random, Bayes, Individual Differences

\section{Introduction}

Is it possible to help people at distance to make the correct choice in a situation where there is no information available to make the correct choice and there are no means of receiving suggestions from someone else using conventional communication channels (i.e. phone call, email, etc.)?

The answer is clearly in the negative if we are referring to a local model of the human mind, stating that the human mind can manifest its contents using only the means available to the human body, i.e. voice and movement. But what we might expect if this model is not correct and the human mind possesses both local and non-local properties, that is human mind may operate without space and time boundaries? The first thing that comes to mind is the possibility of sending information at distance without resorting to the classical means of communication, that is mentally.

Are there theoretical models of non-local mind already available to us and empirical evidence to support them?

The suggestion that the human mind may express non-local properties has its roots in Indian philosophy. In the sacred texts of the Advaita Vedānta philosophical tradition, the physical universe is seen as an undivided whole in which everything is interconnected and the plurality of empirical phenomena is the expression of a unitive and underlying principle of existence and consciousness (mind) called Brahman (Satprakashananda, 2005).

In terms of more recent models of human mind and consciousness, Max Velmans (2009) in his book Understanding Consciousness, developed Reflexive Monism, a philosophical model accounting for the problems of consciousness. This philosophical model is a modern version of an ancient view that the basic stuff of which the universe is composed has the potential to manifest both physically and as conscious experience (a dual-aspect theory in the tradition of Spinoza). Briefly, this philosophical model, posits that in its evolution from some primal undifferentiated state the universe differentiated into distinguishable physical entities, at least some of which have the potential for conscious experience, such as human beings. While remaining embedded within and dependent on the surrounding universe and composed of the same fundamental substance, each human, equipped with perceptual and cognitive systems, has an individual perspective on, both the rest of the universe and him or herself. In this sense, each human participates in a process whereby the universe differentiates into parts and becomes conscious of itself in manifold ways, making the entire process reflexive.

Another theoretical approach to non-local mind is that which sees these properties as quantum-like, similar to the entanglement or interaction at distance phenomena observed in physics (Radin, 2006; Clarke, 1995).

\section{Empirical Evidence}

To summarize the empirical evidence supporting the hypothesis that the human mind possesses non-local properties, we will present data obtained from meta-analysis for a better appreciation of the cumulative evidence available today.

This evidence will be categorized as that investigating psychophysiological responses to non-local interaction between two participants, a sender and a receiver and that investigating the communication of information from a sender to be reported consciously by the receiver.

The more recent meta-analysis of the effects of psychophysiological distant mental interaction between humans is that of Schmidt, Schneider, Utts and Walach (2004), related to two phenomena, the modification of the psychophysiological activiity and the trigger of the feeling of being stared at to a spatially separated and sensory isolated receiver. The synthesis of re- 
spectively 36 and 15 studies reveals a small significant effect size $d=.11$ in the studies on 'direct mental psychophysiological interaction', while a best-evidence-synthesis of 7 studies yielded $d=.05$. In 15 remote staring studies related to the feeling of being stared at, it was obtained a mean effect size of $d=.13$.

What observed with psychophysiological variables has been also observed with electrophysiological measures (Achterberg, Cooke, Richards, Standish, Kozak, \& Lake, 2005; Richards, Kozak, Johnson, \& Standish, 2005; Wackermann, Seiter, Keibel, \& Walach, 2003), but no formal meta-analysis are available at the moment.

A synthesis of evidence related to overt communication at distance is presented by Storm, Tressoldi and Di Risio (2010, under revision). In the prototypical task, a sender tries to connect mentally to a receiver located at distance without any possibility of receiving information by conventional means, in order to send the content of an image or a short video clip. The receiver may be in a Ganzfeld condition or in a normal sensory one. The image or video clip is identified among four, the target and three decoys, after a period of free mentation during which the receiver orally describes all sensory and emotional information or after other techniques she/he adopt to receive the sender information. The summary results obtained with 108 studies using the Ganzfeld technique are: Random Model, ES =.148; .95 $\mathrm{CI}=.098-.198$. The summary results obtained with 14 studies in no altered state of consciousness are: Random Model, ES $=.13 ; .95$ Confidence Interval $(\mathbf{C I})=.07-.19$.

From the cumulative evidence available it seems that mental communication at distance is possible. Theoretical aspects a-part however, one may wonder if this ability is of any practical importance. Measures of effect size do not convey this in formation. From the Ganzfeld database the difference with respect to the Mean Chance Expected (usually .25\%) is: Mean = $10 \%(\mathrm{SD}=.09)$; from the no altered state of consciousness: Mean $=6.5 \%(\mathrm{SD}=7.4 \%)$.

Is a difference of approximately $10 \%$ in a Ganzfeld condition interesting from a practical point of view? The answer is clearly subjective and depends on personal expectation.

Our study is a contribution to increase the difference with respect to the Mean Chance Expected (MCE). To this end, in the first experiment we devised a task that could be perceived as practical by participants. We thought that if participants perceived the task as potentially useful, they would engage more deeply with it and enhance their capacity to send and receive information at distance.

In the second experiment we aimed to explore the role of individual differences testing the role of and Absorption as personality traits and the role of task complexity, as positive moderator variables affecting the efficiency of mental connection at distance.

\section{Experiment 1: Method}

\section{Experimental Design}

Dependent variable: number of correct identification; independent variable: condition of suggestion at distance.

\section{Participants}

A convenient sample of 40 participants was recruited from the students and personnel of Padua University. They were invited to participate in an experiment to study non-local properties of the human mind by a research assistant. The final sample comprised 14 males and 26 females with a mean age of $26 ; \mathrm{SD}=4.3$. For their participation which lasted an average of 30 minutes, they were reimbursed with $€ 5.00$.

\section{Procedure and Material}

The experiment took place in two of the department's labs. Each participant was seated in front of a PC monitor and was informed that the experiment consisted of two sessions and that she/he twice had to recognize 10 real Chinese ideograms and 10 false ideograms. Before starting the experiment, participants were asked about their familiarity with Chinese ideograms. They were enrolled only if they declared no or almost no knowledge apart from the capacity to recognize them as probably Chinese or Japanese ideograms. The choice of this material was dictated by the aim to devise both an attractive task for participants and a task that could not be solved using previous knowledge.

Furthermore, participants were informed that the novelty of the experiment consisted in the possibility of being helped in their decisions by a research assistant who could see the ideograms on a PC located in a distant room by means of an intranet connection. The research assistant could help the participants by suggesting the correct answer at distance but only mentally because no other means of classical communication was possible. To help the receiver, the research assistant used a memo with all the real ideograms printed on it. Although the possibility of being helped at distance was deemed improbable, participants were requested to adopt a positive approach towards this possibility and to answer only after at least 15 seconds of "mental connection" with the helper following their preferred means.

The material (see real and false ideograms in the Appendix) was written by hand by a native Chinese scribe on white paper to get the guarantee about the accuracy of real and false ideograms, scanned and transformed into digital jpg images to be used in the software for automatizing the random presentation of ideograms and the storing of participant' responses. After the presentation of each ideogram in the middle of the PC screen, the participant had to decide if it was real or false, pressing the " $z$ " or the " $m$ " keys on the PC keyboard respectively.

The software was compiled in E-Prime ${ }^{\circledR}$ and the randomization was obtained by using the dedicated subroutine.

In order to prevent learning, no feedback was given after each response. At the end of each session only the number of correct identifications was presented on the PC screen.

To control whether the suggestion at distance could produce better results with respect to a classical situation where only pure intuition could be used, in one of the two sessions the helper was occupied with an irrelevant task and the monitor connected with that of the receiver was turned off. The order of the help sessions alternated among participants but they completed both sessions believing that they had always been helped at distance.

\section{Experiment 1: Results}

In order to test the robustness of the statistical results, we used both a frequentist approach following APA (2010) statistical recommendations and a Bayesian one.

\section{Order of Helping Sessions}

The means of hits of the help sessions according to their order (first or second) were almost identical. No suggestion: First 
session: $\mathrm{M}=10.4$; $\mathrm{SD}=1.9$; Second: $\mathrm{M}=10.5$; $\mathrm{SD}=1.8$; Suggestion at Distance: $\mathrm{M}=11.4 ; \mathrm{SD}=1.7$; Second: $\mathrm{M}=11.2$; $\mathrm{SD}$ $=1.4$. Consequently, they were analyzed together in the following statistical analysis.

\section{Descriptive Statistics}

Means, standard deviation and sum of correct identification (Hits, Min =0; Max =20), are presented in Table 1 .

\section{Comparison with the Mean Chance Expected}

The comparison with the MCE (see Table 2) was done using both a one sample t-test and a binomial test to consider both a continuous and a discrete distribution. For the frequentist approach, all statistics were one-tailed fixed at $\alpha=.05$ and analyzed using a bootstrap procedure based on 1000 samples. For the Bayesian approach, a Bayes Factor $\left(\mathrm{BF}_{10}\right)$ comparing the alternative ver- sus the null hypothesis ratio, was calculated using the software available at

http://pcl.missouri.edu/bayesfactor (Rouder, 2011) for the binomial distribution and the software available at www. ruudwetzels.com/sdtest (Wetzels, Raaijmakers, Jakab, \& Wagen makers, 2009).

\section{Comparison between the Two Conditions}

The statistical comparison between the sessions with and without suggestion at distance is presented in Table 3.

\section{Experiment 1: Comment}

Despite comparison with the MCE, showed that null hypothesis could be rejected for both sessions, if we consider raw scores, effect size and $\mathrm{BF}_{10}$, it is clear that with suggestion at distance, participants obtained the best results. Direct comparison between the two sessions confirms the superiority of suggestion at distance to session without suggestion.

The results seem to support the possibility of sending suggestions at distance without the use of conventional communication means. The net result, however, of a mean increase of $10.3 \%$ with respect to the MCE may seem quite low to be of any practical use.

To test if it were possible to increase the net result with respect to MCE, we decided to carry out a second experiment with more attention paid to individual differences and task complexity.

With respect to individual differences, we concentrated on the degree of Absorption, a particular personality trait that was found favorable to the expression of non-local properties of the human mind (i.e. Terhune and Smith, 2006)

With respect to the task complexity, we realized that the task of suggesting if an ideogram was correct or false is particularly demanding for the helper if she/he is not familiar with the characters. Although we could expect some learning in the course of the experiment, the lack of familiarity meant a continual check of ideograms in the memo, disturbing mental connection with the receiver. To test this hypothesis we devised a simpler task, namely a choice between two pictures representing the sun and the moon respectively (see Appendix).

\section{Experiment 2: Method}

As anticipated, with this experiment we aimed to increase the net result with respect to the MCE taking into account the degree of Absorption as individual difference in personality and the reduction of the complexity of the task as moderating variables.

\section{Experimental Design}

Dependent variable: number of correct identification; independent variable: condition of suggestion at distance.

\section{Participants}

A convenient sample of 70 participants was recruited as in experiment 1 from the students and personnel of Padua University. They were invited to participate in an experiment to study non-local properties of the human mind by a research assistant.

\section{Table 1.}

Means, standard deviation (SD) and sum of correct identification (hits) observed in the sessions with and without suggestion at distance.

\begin{tabular}{ccc}
\hline & No suggestion at distance & Suggestion at distance \\
\hline Mean & 10.55 & 11.33 \\
SD & 1.84 & 1.62 \\
Hits Sum & 422 & 453 \\
\hline
\end{tabular}

Table 2.

Results of frequentist and Bayesian statistics for the sessions without and with suggestion at distance.

\begin{tabular}{|c|c|c|c|c|c|c|}
\hline no suggestion & & One - Sample $\mathrm{t}$ test (.95 CI of difference) & $E S(.95 \mathrm{CI})$ & $\mathrm{BF}_{10}$ & Binomial $\mathrm{z}$ test & $\mathrm{BF}_{10}$ \\
\hline Hits Mean & 10.55 & $\begin{array}{c}1.9 \\
\text { (.5 to } 1.12)\end{array}$ & $\begin{array}{c}.30 \\
(-.2 \text { to } .66)\end{array}$ & 1.31 & 1.52 & 1.3 \\
\hline SD & 1.84 & & & & & \\
\hline \multicolumn{7}{|c|}{ suggestion at distance } \\
\hline Hits Mean & 11.33 & $\begin{array}{c}5.16 \\
(.82 \text { to } 1.85)\end{array}$ & $\begin{array}{c}.81 \\
(.45 \text { to } 1.16)\end{array}$ & 4166 & 3.7 & 23.8 \\
\hline SD & 1.62 & & & & & \\
\hline
\end{tabular}

Table 3.

Frequentist and Bayesian statistics of the comparison between the sessions with and without suggestion at distance.

\begin{tabular}{ccccc}
\hline & & Paired T test (.95 CI of difference) & $E S(.95 \mathrm{CI})$ & $\mathrm{BF}_{10}$ \\
\hline Hits Mean Difference & 0.775 & 2.25 & .44 & 2.6 \\
$\mathrm{SD}$ & 2.17 & $(.15$ to 1.4$)$ & $(.11$ to .76$)$ & \\
\hline
\end{tabular}


The level of Absorption was predefined as low if the total score on the Tellegen Absorption Questionnaire (Tellegen, \& Atkinson, 1974) was at or below 20 and high if it was above 20. An equal number of participants with low and high degree of Absorption was predefined. When the total of 35 was obtained in a group, participants were recruited only if their Absorption level met the inclusion criteria of the remaining group. The final sample comprised 61 males and nine females with a mean age of $27(\mathrm{SD}=3.5)$. For participation lasting an average of 30 minutes, they were reimbursed with $€ 5.00$.

\section{Procedure and Materials}

The procedure was identical to that in first experiment. The only difference was the material. Instead of ideograms, two images were used, a sun and moon. In this case the task of the receiver was to guess which image the helper was suggesting to $\mathrm{him} / \mathrm{her}$. The order of images to be suggested, was randomized creating a list of 0 (sun) and 1 (moon) from the site www.random.org for each participant.

\section{Experiment 2: Results}

\section{Descriptive Statistics}

Means, standard deviation and sum of correct identification (hits, Min $=0, \operatorname{Max}=10$ ) obtained by the Low and High level of Absorption groups, are presented in Table 4.

\section{Direct Comparison between the Two Groups}

The statistics related to the comparison between the two groups of participants are presented in Table 5.

From this comparison it is evident that the two groups performed at similar level and there was no support for a differential ability of one group over the other. Consequently all further analysis were performed only on the whole group.

\section{Comparison with the Mean Chance Expected}

The same frequentist and Bayesian statistics were used as in experiment 1 and are presented in Table 6 .

\section{Experiment 2: Discussion}

The results of experiment 2 converge to support the hypothesis that suggestion at distance was effective with respect to the MCE representing the null hypothesis, with a net increment of $8.8 \%$. The results are no better than those obtained in ex- periment 1 , however, where the net increase with respect to tion MCE was $10.3 \%$. Consequently, our hypothesis that Absorplevel and task simplification could increase performance with respect to the MCE, is not supported.

\section{General Discussion}

Even if they are not extraordinary, our results are close to or better than those obtained with a Ganzfeld procedure suggesting that non-local communication may be observed even without an altered state of sensory perception, clearly not a very practical solution.

The findings of the present study give more support to the hypothesis that the human mind may express non-local properties, both to receive and to transmit information as documented by the meta-analysis of Storm, Tressoldi and Di Risio (2010) and Tressoldi (2011) examining the cumulative evidence obtained up to today.

Although we sometimes described our task from the participants' point of view, what we observe may be not a transmission of information but rather only correlations between two entangled minds. What some philosophical models and Quantum Mechanics interpretations tell us, is that deep physical reality is interconnected beyond the ordinary boundaries of space and time that in turn tells us that our minds/consciousness must also be interconnected. Not occasionally, but always.

If this is true, then what we observe is our experiential, subjective sense of this underlying connectedness. Therefore suggestion at distance is not transmission of anything. It may be, rather, a result of focusing one's attention on another person, because we are already and continuously connected to that person all the time (and to everything else). By analogy, if we had a radio that transmitted everyone's speech in the universe to us simultaneously, all we would hear would be noise. But if we tuned the radio to one specific frequency, we would hear one specific person. We think that mental connection at distance is the act of attention tuning the radio.

Table 4.

Means, standard deviation (SD) and sum of correct identification (hits) of participant with low and high level of absorption.

\begin{tabular}{cccc}
\hline Groups & Mean & SD & Hits Sum \\
\hline Low & 5.37 & 1.78 & 188 \\
High & 5.51 & 1.48 & 193 \\
All & 5.44 & 1.63 & 381 \\
\hline
\end{tabular}

Table 5 .

Frequentist and Bayesian statistics related to the comparison between the low and high absorption groups.

\begin{tabular}{lccc}
\hline & One sample t-test (.95 CI of the difference) & $E S(.95 \mathrm{CI})$ & $\mathrm{BF} 10$ \\
\hline Hits Mean Difference & .14 & $(-.88$ to .60$)$ & .08 \\
\hline
\end{tabular}


Even if there is some hypothesis, there is a lack of knowl edge about the moderating factors influencing the performance of non-local mental connection. As far as individual differences are concerned, there is insufficient information about the role of some personality traits. In experiment 2 , we failed to identify the role of Absorption level. Among other personality traits, there is the Sensation-Seeking one. Recently, this trait has been observed as a positive mediator in tasks requiring participants to anticipate future information (Bem, 2011). In general, the search for a good personality trait should consider those which favour mental and emotional bonding (Miller and Rodgers, 2001), but little is known about these at the moment.

A third variable that could positively moderate non-local mental connection, may be the degree of emotional bonding between the sender and the receiver. Sheldrake and colleagues for example, observed an increase of correct hits when the sender was a parent or a close friend in task where the receiver had to guess who had rung or emailed them (Sheldrake and Smart, 2005; Sheldrake and Beharee, 2009). A plausible explanation is that people who shared emotional and communication bonds for long time, find communication easier whether using classical or non-local means.

Although the evidence supporting the reality of non-local perception and communication abilities of the human mind is quite robust, these abilities are only likely to be appreciated by lay persons and scientists alike, if demonstrations of their practical use are provided. Our study is a contribution towards this goal and although our findings are not particularly exceptional, we hope more people will become interested in this research field to contribute to enlarging our knowledge of the human mind.

\section{Acknowledgements}

We acknowledge the Proof Reading Service for English revision and the suggestions of the referee which helped us to improve the clarity of the paper.

\section{References}

Achterberg, E., Cooke, K., Richards, T., Standish, L. J., Kozak, L., \& Lake, J. (2005). Evidence for correlations between distant intentionality and brain function in recipients: A functional magnetic resonance imaging analysis. Journal of Complementary and Alternative Medicine, 11, 965-971. doi:/10.1089/acm.2005.11.965

American Psychological Association. (2010). The publication manual of the American psychological association (6th ed.). Washington, DC.

Bem, D. J. (2011). Feeling the future: Experimental evidence for anomalous retroactive influences on cognition and affect. Journal of Personality and Social Psychology, 100, 1-19. doi:/10.1037/a0021524

Clarke, C. J. S. (1995). The non-locality of mind. Journal of Consciousness Studies, 2, 231-240.
Miller, W. B., \& Rodgers, J. L. (2001). The ontogeny of human bonding systems: Evolutionary origins, neural bases, and psychological manifestations. New York: Springer.

Radin, D. (2006). Entangled minds: Extrasensory experiences in a quantum reality. New York: Paraview Pocket Books.

Richards, T. L., Kozak, L., Johnson, L. C., \& Standish, L. J. (2005). Replicable functional magnetic resonance imaging evidence of correlated brain signals between physically and sensory isolated subjects. Journal of Complementary and Alternative Medicine, 11, 955-963. doi:/10.1089/acm.2005.11.955

Rouder, J. N. (2011). Bayes factor for a binomially distributed observation. http://pcl.missouri.edu/bfbinomial

Rouder, J. N., Speckman, P. L., Sun, D., Morey, R. D., \& Iverson, G. (2009). Bayesian t tests for accepting and rejecting the null hypothesis. Psychonomic Bulletin \& Review, 16, 225-237. doi:/10.3758/PBR.16.2.225

Satprakashananda, S. (2005). Methods of knowledge according to advaita vedānta. Kolkata: Advaita Ashrama.

Schmidt, S., Schneider, R., Utts, J., \& Walach, H. (2004). Distant intentionality and the feeling of being stared at: Two meta-analyses. British Journal of Psychology, 95, 235-247.

doi:/10.1348/000712604773952449

Sheldrake, R., \& Beharee, A. (2009). A rapid online telepathy test. Psychological Reports, 104, 957-970. doi:/10.2466/pro.104.3.957-970

Sheldrake, R. \& Smart, P. (2005). Testing for telepathy in connection with e-mails. Perceptual and Motor Skills, 101, 771-786.

Storm, L., Tressoldi, P. E., \& Di Risio, L. (2010). Meta-analysis of free-response studies, 1992-2008: assessing the noise reduction model in parapsychology. Psychological Bulletin, 136, 471-485. doi:/10.1037/a0019457

Storm, L., Tressoldi, P. E., and Di Risio, L. (under revision). Metaanalysis of ESP studies, 1987-2010, assessing the success of the forced-choice design in parapsychology.

Tellegen, A., \& Atkinson, G. (1974). Openness to absorbing and selfaltering experiences ("absorption"), a trait related to hypnotic susceptibility. Journal of Abnormal Psychology, 83, 268-277. doi: $/ 10.1037 / \mathrm{h} 0036681$

Terhune, D. B. \& Smith, M. (2006). The Induction of anomalous experiences in a mirror-gazing facility: suggestion, cognitive perceptual personality traits and phenomenological state effects. Journal of Nervous \& Mental Disease, 194, 415-421. doi:/10.1097/01.nmd.0000221318.30692.a5

Tressoldi, P. E. (2011). Extraordinary claims require extraordinary evidence: The case of non-local perception, a classical and Bayesian review of evidences. Frontiers in Psychology, 2, 1-5. doi:/10.3389/fpsyg. 2011.00117

Velmans, M. (2009) Understanding consciousness (2nd ed.). London: Taylor \& Francis.

Wackermann, J., Seiter, C., Keibel, H., \& Walach, H. (2003). Correlations between brain electrical activities of spatially separated human beings. Neuroscience Letters, 336, 60-64. doi:/10.1016/S0304-3940(02)01248-X

Wetzels, R., Raaijmakers, J. G. W., Jakab, E., \& Wagenmakers, E. J. (2009). How to quantify support for and against the null hypothesis: A flexible WinBUGS implementation of a default Bayesian $t$ test. Psychonomic Bulletin \& Review, 16, 752-760. doi:/10.3758/PBR.16.4.752 


\section{Appendix}

Ideograms used in experiment 1

Real:

$7=$<smiles>C1CCCCC1</smiles>

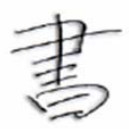

六妨

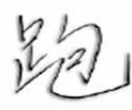

$\int \frac{\pi}{2}$<smiles>CC(C)C(C)(C)C(C)(C)C</smiles>

产 $\quad \sqrt{k}$
False:

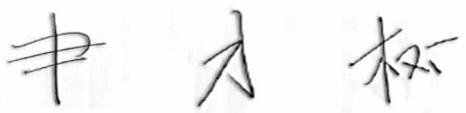

in

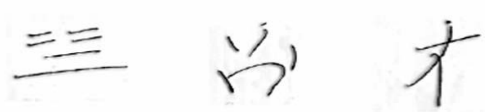

37

Images used in experiment 2

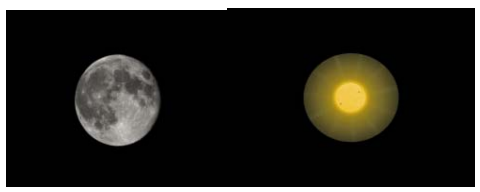

